\title{
25 \\ TO REPEAT OR NOT TO REPEAT: FACE-ING THE FACTS
}

\author{
Michael Burmester, Werner Beck, Paul Hubert Vossen \\ Fraunhofer-Institut für Arbeitswirtschaft und Organisation \\ Nobelstraße 12, 70569 Stuttgart, F.R. Germany
}

Phone +49 7119702311, Fax +49 7119702300

E-mail: Michael.Burmester@iao.fhg.de

KEYWORDS: auto-repeat, repeated function, user interface design, rapid data input

\begin{abstract}
Auto-repeat is the automatic repetition of a function by continuous pressing of a button. In three separate tests, the design and operation of this auto-repeat feature for various electronical devices was examined. Eleven subjects took part in the experiment. Each subject was given the same stratgies for optimizing the auto-repeat feature. Both performance variables (ie. task completion time, number of key strokes) and attitude variables (task difficulty, task effectiveness, task satisfaction) were employed. The results show that optimization of the auto-repeat feature in case of larger distances between origin and target is not only sensible but also desirable: three phases when varying repeat delay time, or two phases when varying repeat step size.
\end{abstract}

1

\section{INTRODUCTION}

Auto-repeat denotes the automatic repetition of a function through continued activation of a control for setting numerical values. The auto-repeat function appears in various user realms (ie. the setting of a clock, selecting a channel on a TV set, setting a counter value, etc.).

From a scientific point of view, in spite of its frequent use, the auto-repeat function appears to be a neglected topic (see e.g. Potosnak, 1988).

In the framework of the FACE project ${ }^{1}$ (Burmester \& Machate, 1994) the issue of both efficient and effective optimization of the auto-repeat function receives a new pragmatic background. The idea behind FACE (Familiarity Achieved through Common User Interface Elements) is to define, develop and make available rules and guidelines for the design of uniform control elements and dialogue concepts for as many electronic appliances in the home as possible (ie. radio, TV, video recorder, telephone, washing machine etc.).

In trying to improve the keyboard functions for data input both economically and ergonomically, the issue of the optimization of the auto-repeat function came about.

\section{A MODEL OF AUTO-REPEAT AND ASSOCIATED RESEARCH QUESTIONS}

\subsection{Parameters of auto-repeat}

Three of the most important parameters of the autorepeat function were studied: the repeat-step size (RSS), the repeat-delay time (RDT), and the number of distinct repeat phases.

Initial-delay and repeat-delay time $\mathrm{T}$ he initial delay time is the time span between the activation (ie. pressing) of e.g. a PLUS or MINUS button and the start of the auto-repeat function. Repeat delay time is defined as the time lag until a value is changed again by the system.

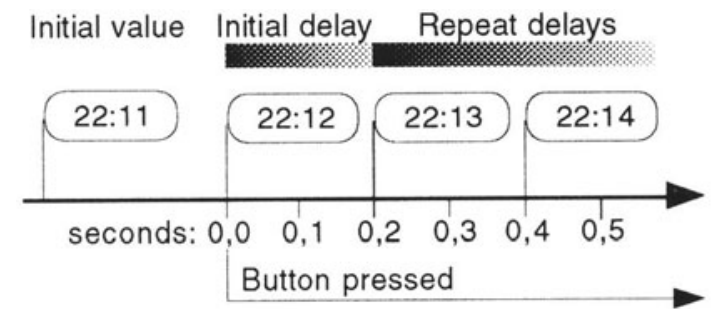

Figure 1: The basic concepts of auto-repeat 
Example Initial delay and repeat delay time do not necessarily have the same value, but in the preceding figure the value for both parameters is 0.2 seconds. Once the user has pressed say a dedicated button for clock setting, the displayed time repeatedly changes by a unit of one minute on the digital clock. This takes place after a constant delay of 0.2 seconds as long as the user keeps pressing this button.

Repeat-step The repeat-step size is defined as the counting unit added to or subtracted from the current value of some counter, e.g. a clock.

Example An increment of 10 used for setting a counter, ie. on a VCR. When the user presses and holds down a dedicated counter setting button, the counter value on the display changes by a unit of 10 :

initial value: 124

press PLUS: 125 keystroke step size is 1

hold PLUS: 135 auto-repeat step size is 10

hold PLUS: 145 auto-repeat step size is 10

\subsection{Optimization model}

The simplest auto-repeat function has a constant repeat-step size (RSS), a constant repeat-delay time (RDT), and no acceleration phases. In the sequel, this is denoted as the 1-phase model, where RSS $=1$ and $\mathrm{RDT}=0.2$ seconds, constantly.

A phase-wise decrease in repeat-delay time In accordance with standard ergonomic recommendations also adopted by the FACE project, the following models were defined (note that a RDT of 0.08 represents the highest possible speed of the PC used in this experiment):

\section{2-Phase Model $(\mathrm{RSS}=1)$ :}

Phase $1 \quad$ : RDT $=0.20 \mathrm{~s}$

Phase 2 (after $10 \times$ Phase 1) : RDT $=0.10 \mathrm{~s}$

3-Phase Model ( $\mathrm{RSS}=1)$ :

Phase $1 \quad:$ RDT $=0.20 \mathrm{~s}$

Phase 2 (after $10 \times$ Phase 1) : RDT $=0.10 \mathrm{~s}$

Phase 3 (after $20 \times$ Phase 2) : RDT $=0.08 \mathrm{~s}$

Phase-wise increase of the repeat-step size For the purpose of this experiment, two different models dealing with the increase of the repeat-step size (numeral/time) are defined:

\author{
2-Phase Model (RDT $=0.2 \mathrm{~s}$ ) \\ Phase $1 \quad: \quad \mathrm{RSS}=1 ; 1 \mathrm{~m}$ \\ Phase 2 (after $10 \times$ Phase 1) : $\quad$ RSS $=10 ; 10 \mathrm{~m}$ \\ 3-Phase Model (RDT $=0.2 \mathrm{~s}$ ) \\ Phase $1 \quad$ : $R S S=1 ; 1 \mathrm{~m}$ \\ Phase 2 (after $10 \times$ Phase 1) : $\quad$ RSS $=10 ; 10 \mathrm{~m}$ \\ Phase 3 (after $10 \times$ Phase 2) : RSS $=100 ; 1$ h
}

\subsection{Research Questions}

The following research questions have been tested:

- Does a phase-wise decrease of the repeat-delay time lead to an improvement of the usability of the auto-repeat function? If so, with which kind of model (2-phase or 3-phase)?

- Does a phase-wise increase of the repeat-step size lead to an improvement of the usability of the auto-repeat function?

- Does an speed indicator providing feedback on the current repeat phase result in a better handling of a phase-wise reduction of RDT?

\section{METHOD}

\subsection{Sample}

The sample consisted of 6 female and 5 male test subjects ranging in age from 23 to 35 years. The average age was a little above 28 years. Most of the participants (8) were students.

\subsection{Independent Variables}

A decrease of repeat-delay time as well as an increase in the repeat-step size have been selected as independent variables. Both variables have been varied threefold: 1-phase model, 2-phase model, 3-phase model.

As an additional independent variable, the efficiency of the speed indicator of repeat-delay time with the 2phase and 3-phase models of the decrease in repeatdelay time has been tested (ie. two levels: one with and one without a speed indicator).

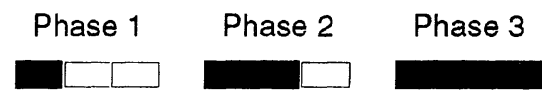

Figure 2: The speed indicator 


\subsection{Dependent Variables}

Two empirical variables (execution time for each task, number of keystrokes) and three attitude variables (estimating task difficulty, estimating task effectiveness and satisfaction with the method of entering the desired value) were used as dependent variables.

The three attitude variables were measured on a 5point Lickert scale ranging from -2 (least negative answer) to +2 (most positive answer).

\subsection{Task Options}

Various task options (2-digits: 00-99, 3-digits: 000999 , a clock in 24-hour digital format) and different intervals between the start and stop values to be entered were used as control variables (for each type of display: larger intervals vs. smaller intervals, keeping them constant within the various task options). In order to avoid positioning effects, the sequence of items for the various levels of the variables were fully balanced out or randomly selected.

\subsection{Test Designs}

A test design was developed for each of the three independent variables:

- Phase-wise decrease of the repeat-delay time

- Phase-wise increase of the repeat-step size

- Speed indicator absent or present

Phase-wise decrease of repeat-delay time and phasewise increase of repeat-step size had a very similar design. All of the display types (2-digit numbers, 3digit numbers, $24 \mathrm{~h}$ clock) resulted in three levels of RSS or RDT (constant auto-repeat, 2-phase optimization, or 3-phase optimization). Each subject was tested with all three levels of both factors as well as with each display type.

The sequence of the three models was balanced out for each task option and resulted in 6 sequences. The sequence of task options was held constant. Two tasks were carried out for each combination of a task option and a RSS or RDT level: one task having a larger interval between start and target values and another one with a smaller interval.

Thus, e.g., for the assessment of the influence of repeat-delay time, two "1-phase" items, two "2phase" items and two "3-phase" items were used. A classical experimental control group design was used for the speed indicator variable.

\subsection{Experimental Procedure}

-Each of the subjects were seated in front of a monitor which was hooked up to a PC (i80486 CPU) via a VGA-card. The display indicating the values to be changed and the controls to be used (a PLUS and a MINUS button) were simulated ${ }^{2}$ on the monitor.

Each task was displayed at the start of each test. The subjects were given a start value along with a target value to be attained by the pressing of virtual PLUS and MINUS buttons using the mouse.

At first, the test subjects were made familiar with the test situation through the employment of 6 practice tasks. Following this, they were randomly given six tasks with 18 test items, six tasks with 16 test items and six tasks with 12 test items for the evaluation of the decrease of repeat-delay time, for the increase in repeat-step size and the settings of the speed indicators, respectively.

\section{RESULTS}

For the evaluation of each of the following results a one-factorial analysis of variance with repeated measures was used.

\subsection{Effect of Repeat-Delay Time}

There is no evidence for a decrease in task completion time due to a phase-wise decrease of RDT in the case of a display with two digits. Actually, the task completion times increase significantly in both multiple-phase models (see Table 1).

With a 3-digit display as well as with a time display, the 3-phase decrease of repeat-delay time (RDT = $0.2 \mathrm{~s}, 0.1 \mathrm{~s}, 0.08 \mathrm{~s}$ ) shows a very significant lower task completion time than the 1-phase model (RDT $=0.2 \mathrm{~s}$ ) or the 2-phase model (RDT $=0.2 \mathrm{~s}, 0.1 \mathrm{~s}$ ). With this scenario, the number of key strokes does not increase significantly. The level of satisfaction also gives empirical evidence for the superiority of the 3-phase model.

The not so impressive results of the 2-phase model were rather astonishing. Compared with the 1-phase model, the 2-phase-model does not show any superiority on any usability dimension.

Conclusion In case of an optimization of the auto-repeat function by a reduction of the repeat delay time, a 3-phase model is recommended. Beside offering a lower task completion time with a slight increase in the number of keystrokes, the 3-phase model offers significantly favorable usability values. 
Table 1: Results of a one-factor ANOVA with repeated measures to test the effect of varying repeat-delay times with a constant repeat-step size of 1

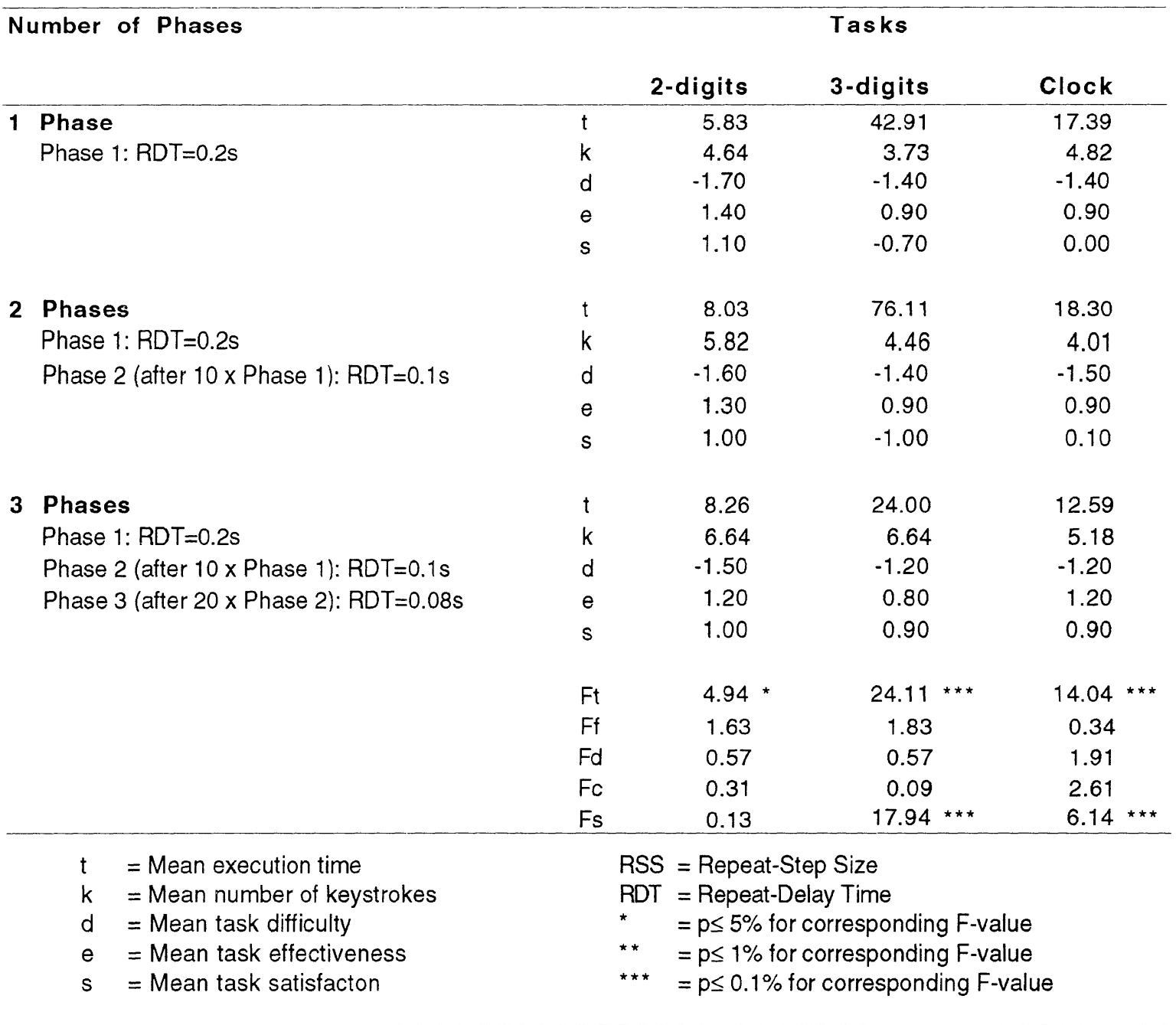

\subsection{Effect of Repeat-Step Size}

The results show that an optimization of the autorepeat function with a stage-wise increase in increments is sensible only in bridging the gap between larger intervals.

A negative effect was shown in dealing with 2-digit numbers. Here, although the increase of task completion time is not significant, a clear tendency to increase (1-Phase: 7.1s; 2-Phase: 10.11s) can be observed. At the same time, the number of keystrokes increases significantly (see Table 2 ).
Also, there is a tendency to assess the subjective task difficulty higher when there is a change in the repeat-step size. The judgement of the task effectiveness is significantly higher without incremental changes. The harm done in using a phase-wise increase of the repeat-step size is smaller with 2-digit figure displays. The acceptance of the alleged optimization is however greater.

With 3-digit displays and large setting intervals, the task completion time shows a significant effect for a step-wise increment in the repeat-step size. The 3step model $(\mathrm{RSS}=1, \mathrm{RSS}=10, \mathrm{RSS}=100)$ does not cause a significant reduction in the processing time as is with the 2-step concept. The 2-step as well as 
Table 2: Results of a one-factor ANOVA with repeated measures to test the effect of varying repeat-step size with a constant repeat-delay times of 0.2 seconds

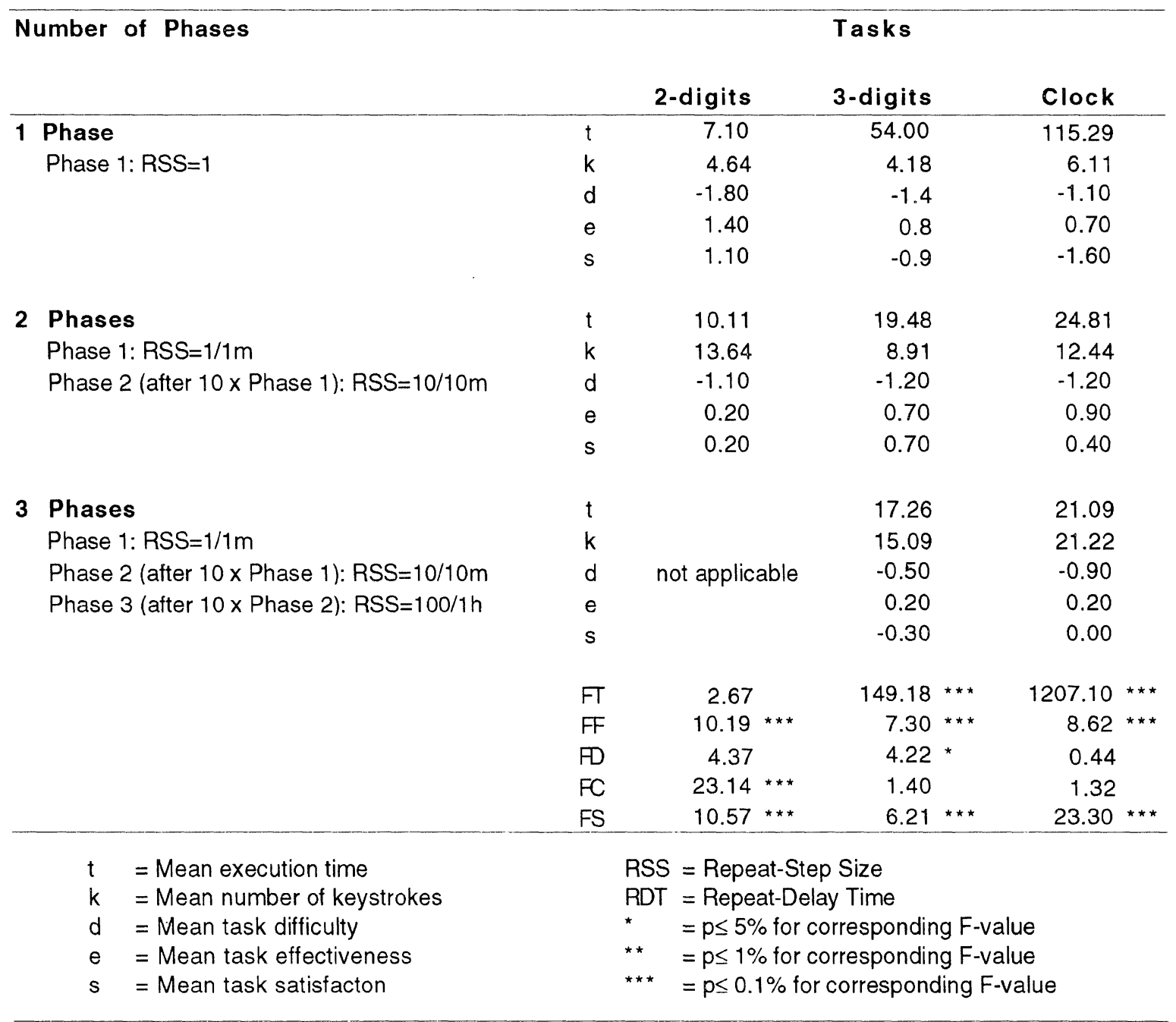

the 3-step change in the increment shows a highly significant advantage in comparison to the constant increment (3-Phase:s: 17.26s, 2-Phases: 19.48s, 1Phase: 54.1s).

At the same time, the number of keystrokes increases significantly (3-Phases: 15.09, 2-Phases: 8.91 , 1-Phase 4.18)

The subjective satisfaction shows a similar significant effect: the 1-Phase model is least favored, the 2-Phase model is most lavored, with the 3-Phase model in between.
In setting the time of a clock having a 24 hour time format, similar results were shown as with the 3digit number display.

Conclusion If the auto-repeat function is to be optimized through an enlargement of the step size, - which can be very helpful in dealing with large intervals between starting and target values - then the 2-Phase model ( $R S S=1 ; \operatorname{RSS}=10$ ) is the most suitable.The 2-Phase model has the best results regarding task completion time, reasonable number of keystrokes and overall satisfaction in case of large to very large intervals. 


\subsection{Effect of Speed Display Feedback}

No significant effect was found here. Also, the subjective opinion found in the interviews taken at the end of the testing were very dispersed.

DISCUSSION

The goal of this experiment was to test under which precise conditions the use of an auto-repeat feature is sensible and preferable. These conditions were:

(1) varying repeat-delay times

(2) varying repeat-step sizes and

(3) varying number of acceleration phases.

As soon as the setting of values having intervals greater than say 50 units takes place, the optimization in form of a decreasing repeat-delay time or an increasing repeat-step size appears to be sensible. On the basis of our test results, a 3-phase model with decreasing repeat-delay times (Phase $1:$ RDT $=0.2 \mathrm{~s}$ Phase 2 after 10 times Phase 1: RDT $=0.1 \mathrm{~s}$, Phase 3 after 20 times Phase 2: RDT $=0.08 \mathrm{~s}$ ) is recommended. The 3-phase model achieves favorable task completion times and high usability values with only a moderate increase in the number of keystrokes.

A 2-phase model with increasing repeat-step size (Phase 1: RDT $=0.2, \mathrm{RSS}=1$ after 10 times Phase 1: $\mathrm{RSS}=10$ ) is effective in decreasing the task completion time with larger intervals, but has the opposite effect when dealing with smaller intervals. It should be noted that with an increase in the repeat-step size, more keystrokes are necessary, because even theoretically the end value can not be reached with a single keystroke.

The cognitive expenditure seems to be higher with a model having varying repeat-step sizes. With very large intervals, the factor of task completion time earns a new meaning. With that the user wants to have an option which can not be provided by a 3phase model of decrementing the repeat delay time. Eventually, a combination of the favored 3-phase model (reduction in repeat-delay time) and the favored 2-phase model (increase of the increment) could be the optimal solution to the problem. Our modified 3-phase model is as follows:

\section{Phase 1}

$: \mathrm{RDT}=0.2 \mathrm{~s} / \mathrm{RSS}=1$

Phase $2($ after $10 \times$ Phase 1$):$ RDT $=0.1 \mathrm{~s} / \mathrm{RSS}=1$

Phase 3 (after $20 \times$ Phase 2$):$ RDT $=0.1 \mathrm{~s} / \mathrm{RSS}=10$
Theoretically, this will make it possible to combine the advantages of the repeat-step size increase, which is very effective in working with larger intervals (over say 50 units), with the advantages of a decrease in repeat-delay time with smaller intervals (less than say 20 units).

The question, whether this 3-phase model or possibly a smooth change in both repeat-step size or repeat-delay time, can allow for a still more favorable optimization of the auto-repeat feature is a question to be answered in further research work.

\section{ACKNOWLEDGEMENTS}

We like to thank Nick Shepherd for suggesting the idea for this experiment and for building the prototyping tool. Furthermore we gratefully acknowledge the excellent support of Hans-Georg Scheller a first pilot experiment (not reported here).

\section{REFERENCES}

Burmester, M. \& Machate, J. (1994): "Common User Access" for Electronic Home Devices or 20 Ways to Set the Clock, in: Proceedings of the ECCE7, Bonn FRG, pp. 97-116,

Potosnak K.M. (1988), Keys and keyboards, in: Helander, M. (Ed.) : Handbook of Human-Computer Interaction. Amsterdam: North Holland, Chapter 21, pp. 475-494

RAPRO-T (1992-94): Rapid Prototyping Tools. Philips Consumer Electronics, Advanced Projects Group

\section{NOTES}

(1) The FACE consortium was composed of four major European electronic domestic appliance companies (Philips CE APG, UK; Thomson CE\&CSF, France; Legrand, France; Bticino, Italy) and two human factors research institutes (Stuttgart University IAT, Germany and Loughborough University HUSAT, UK).

(2) The test scenario was developed with the user interface prototyping and simulating tool RAPRO-T from Philips Consumer Electronic Company. 